\title{
Modernités dans les Amériques : des avant-gardes à aujourd'hui
}

Fiona McMahon et Paul-Henri Giraud

\section{OpenEdition}

Journals

Édition électronique

URL : https://journals.openedition.org/ideas/2544

DOI : $10.4000 /$ ideas.2544

ISSN : 1950-5701

Traduction(s) :

Modernities in the Americas: from the avant-gardes to nowadays - URL : https:// journals.openedition.org/ideas/3125 [en]

Modernidades en las Américas: de las vanguardias a la actualidad - URL : https:// journals.openedition.org/ideas/3131 [es]

As modernidades nas Américas: desde as vanguardas até hoje - URL : https:// journals.openedition.org/ideas/3151 [pt]

Éditeur

Institut des Amériques

\section{Référence électronique}

Fiona McMahon et Paul-Henri Giraud, « Modernités dans les Amériques : des avant-gardes à aujourd'hui », IdeAs [En ligne], 11 | 2018, mis en ligne le 19 juin 2018, consulté le 20 octobre 2022 URL : http://journals.openedition.org/ideas/2544 ; DOI : https://doi.org/10.4000/ideas.2544

Ce document a été généré automatiquement le 20 octobre 2022.

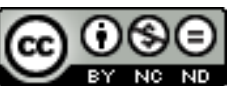

Creative Commons - Attribution - Pas d'Utilisation Commerciale - Pas de Modification 4.0 International - CC BY-NC-ND 4.0

https://creativecommons.org/licenses/by-nc-nd/4.0/ 


\title{
Modernités dans les Amériques : des avant-gardes à aujourd'hui
}

\author{
Fiona McMahon et Paul-Henri Giraud
}

1 Reposer aujourd'hui la question de la modernité - littéraire, artistique, sociale ou politique - revient à employer le mot au pluriel. Ce choix implique que l'on ne se réduise pas au terme plurivoque, et souvent ambigu, de modernisme. Ce dernier, souvent convoqué pour désigner l'imaginaire culturel de la modernité, peine à rendre compte des écarts qui se manifestent dans la manière de penser le rapport au temps, à l'histoire et à la culture. "La modernité n'est pas un mouvement, comme dada ou l'imagisme. Si l'histoire littéraire décide de dénommer modernisme tel mouvement, anglais ou espagnol, aussitôt le terme prend un sens technique. Il se fixe. Il ne participe plus que fragmentairement de la modernité " (Meschonnic H., 1988:26). Le concept de modernité, dans le sillage de ses variations européennes révélées au seuil du vingtième siècle - technologiques, politiques, artistiques -, est tout autant marqué par la disparité des expériences dans les lieux et les espaces des Amériques. Le présent numéro de la revue IdeAs cherche à mettre en perspective ce concept dans le domaine des arts et de la littérature, à partir du moment clé des avant-gardes nées de la Première Guerre mondiale. Quels sont, dans les Amériques, les retentissements des bouleversements culturels introduits en Europe et, à l'inverse, quelles formes de résistance viennent éclairer les productions américaines?

2 Il s'agit donc d'envisager à nouveaux frais un phénomène multiforme et multilingue, afin de dégager des lignes de force ou de partage. Pour les différentes aires culturelles des Amériques, quels horizons sont offerts par le paradigme à la fois destructeur et créateur de la modernité ? «Au nom de la radicalité artistique et du concept de rupture ", on "a laissé de côté ou minoré de nombreuses expressions individuelles ou collectives jugées hybrides, locales, tardives ou antimodernes ", peut-on lire au sujet de l'art (Grenier C., 2013 : 16). De même, en littérature, c'est au modèle de l'éternel avenir du passé que l'on continue à se mesurer: "il faut être ancien pour avoir quelque chance d'être moderne ou de décréter la modernité » (Casanova P., 2008: 137). De là la recherche systématique d'ancêtres du moderne jusque dans les mondes dits 
«primitifs » ou indigènes. Pour reprendre et inverser l'antithèse classique posée par l'Argentin José Domingo Sarmiento (2006) au milieu du XIX ${ }^{e}$ siècle, souvent la «barbarie » paraît plus moderne que la «civilisation", du moment que l'on sait se garder de la tentation folkloriste - ce à quoi s'emploient les stylisations du moderne quand elles s'appliquent au matériau vernaculaire.

3 Les différences indéniables autant que les points de convergence entre les Amériques anglophone et hispanophone - en regrettant que les domaines lusophone et francophone n'aient pas pu être représentés dans ce numéro - devraient permettre de mieux comprendre les spécificités de chacune de ces régions et leurs propres contrastes internes, depuis les premières décennies $d u X X^{e}$ siècle jusqu'à la période actuelle. Car c'est seulement aujourd'hui que les modernités successives trouvent à la fois leur forme exacte et leur réponse, tant dans les remises en cause opérées par les pensées postcoloniale ou décoloniale que dans certains prolongements des arts, de la littérature, voire de l'ethnologie.

\section{Peinture, photographie et danse}

4 L'avant-garde du début du XX ${ }^{e}$ siècle, marquée par la succession des "-ismes", développa activement l'utopie de mêler et de faire converger les arts. Au Mexique, dans le bouillonnement culturel qui suivit la Révolution, littérature, peinture, gravure, photographie et calligrammes se retrouvent dans la revue Irradiador (1923), laquelle se définit, dans son sous-titre, comme «Revue d'avant-garde, projecteur international d'esthétique nouvelle». Le culte du nouveau, le rejet agressif de l'ancien, la mise en valeur esthétique de l'arbitraire et du fragmentaire, la poésie chaotique de la ville moderne, selon Isabelle Pouzet, définissent l'essence de l'éphémère mouvement "stridentiste" dont cette revue se fit le porte-voix. En couverture du troisième numéro, une photographie d'Edward Weston prise en 1922 dans l'Ohio, intitulée Steel, présente une rangée de cheminées d'usine en contre-plongée. Cette introduction détonante d'une image brute et stylisée de l'industrie états-unienne en première page d'Irradiador marque l'avènement au Mexique de la Straight Photography, et manifeste la puissante aspiration à la modernité des artistes de ce pays, sous l'impulsion du grand voisin du Nord. Le séjour de Weston au Mexique entre 1923 et 1926 fait ainsi le trait d'union entre les avant-gardes du nord et du sud du Río Grande.

5 À l'euphorie iconoclaste d'Irradiador, dont l'enthousiasme militant et œcuménique pourrait presque être qualifié d'ingénu, fit suite, avec l'institutionnalisation de la Révolution mexicaine, une utilisation plus critique du médium photographique. Contre la "mythologie d'un modernisme triomphant ", en décalage avec la "mise en scène nationaliste dans la culture visuelle " que la peinture muraliste ou la sculpture à la gloire du régime mirent en œuvre pendant les années 1920-1930, Erica Segre discerne dans la photographie mexicaine contemporaine, et notamment chez Agustín Jiménez, un «courant réflexif tourmenté par le cadrage et le pouvoir coercitif profond». Elle s'attache à mettre en valeur les « esthétiques dissidentes » qui, par le jeu des ombres et des grilles, suggèrent l'enfermement des Mexicains dans un unanimisme national. D'abord souterraine, cette sourde « indiscipline " photographique ne devient évidente que dans les années 1950-1960, dans les photos de Nacho López, Rodrigo Moya ou encore Héctor García. Les portraits réalisés par ce dernier du peintre David Alfaro Siqueiros (1896-1974) derrière les barreaux de la prison de Lecumberri, au début des 
années 1960, font de cet artiste marxiste une véritable icône. Rester moderne, pour Siqueiros, impliqua de pouvoir circuler entre les statuts antinomiques de peintre d'avant-garde, de peintre officiel et de paria, à travers la performance interrompue de sa geste artistico-politique. Le muraliste qui ne cessa de rejeter la peinture de chevalet parvint, malgré tout, à ne jamais sortir du « cadre » médiatique, et ce grâce au cadrage photographique.

6 Son contemporain états-unien, Stuart Davis (1892-1964), offre un cas très différent. Kamila Benayada voit dans son travail une « redéfinition du modernisme " à travers "la série des Champion, peinte pendant la Guerre Froide ». Si la construction de l'œuvre d'art est axée sur la relation qu'elle entretient avec le monde, la pratique de Davis consiste à complexifier les termes de cette relation en créant un dialogue avec ses propres œuvres antérieures tout comme avec celles de ses prédécesseurs modernistes, et en élargissant la sphère artistique à l'environnement visuel de la culture populaire. La transitivité de l'œuvre peinte rattache les gestes picturaux au monde et à ses contextes sémiotiques. Rendu visible à la surface de la toile, le rapport de l'artiste à son temps sous-tend un dispositif d'invention picturale qui, faisant fi des frontières génériques, célèbre le mouvement et l'échange.

7 Elsa Crousier, pour sa part, retrace l'évolution de la critique d'art d'origine uruguayenne Marta Traba, qui fonda le Musée d'Art Moderne de Bogotá en 1963. Après avoir tenu un discours internationaliste et cosmopolite sur l'art, Traba s'attacha, à partir des années 1960, à définir l'art latino-américain contre le Nord - l'Europe, mais plus encore les États-Unis. La « résistance » latino-américaine passait, selon elle, par un rejet des poncifs superficiellement modernes du Pop Art et par une plongée dans l'identité régionale et la psyché amérindienne. Pour être modernes, les artistes latinoaméricains devaient donc, d'une certaine manière, se faire anti-modernes.

8 De même, on peut observer, avec Claudie Servian, que la modernité et le décentrement culturel auquel elle donne lieu aboutissent à une métamorphose du paysage chorégraphique états-unien dans le premier vingtième siècle. Dans les écrits théoriques des chorégraphes Martha Graham (1894-1991) et Doris Humphrey (1895-1958) ici analysés, la quête de la modernité implique une mise au jour du passé le plus ancien. Le fantasme de l'origine, doublé d'un rejet du modernisme technologique, conduit la danse vers l'exploration d'archaïsmes perdus, enfouis dans l'inconscient ou identifiés aux modèles empruntés aux cultures amérindiennes. Le retour aux arts tribaux et au psychisme primitif oppose le mécanisme de la modernité aux mouvements du corps dans ce qu'ils ont d'intuitif et de naturel. La modernité du corps tiendrait donc à son potentiel expressif et signifiant, dans une relation de contact ou de communion avec son environnement. L'interaction avec le sol serait le gage d'une création authentique, en même temps qu'elle fait surgir les décors de l'histoire américaine, comme dans certaines chorégraphies des années 1930. Ainsi, la rupture avec la tradition académique européenne passe par le mythe d'un retour aux sources.

On observe un mouvement semblable en Amérique latine dans les années 1960 autour de la notion de néo-baroque, ici étudiée par Marcos Rico Domínguez. L'art colonial hispano-américain opéra la synthèse des civilisations préhispaniques et du catholicisme espagnol. La catégorie esthétique de baroque, inventée à la fin du XIX ${ }^{\mathrm{e}}$ siècle mais surtout théorisée dans la première partie du $\mathrm{XX}^{\mathrm{e}}$ siècle, fut revendiquée dans les années 1960 par une constellation d'auteurs cubains, et analysée par le Mexicain Octavio Paz (1914-1998) comme l'un des fondements de la modernité latino- 
américaine. Paz opérait ainsi la jonction entre l'époque historique dite "moderne " (XVI ${ }^{\mathrm{e}} \mathrm{XVIII}{ }^{\mathrm{e}}$ siècles), apogée de l'Empire hispanique, riche de nombreuses semences, et la déconcertante prolifération des signes dans l'avant-garde des années 1960, en mettant en valeur le substrat indigène d'un art en apparence très occidental et " moderne ».

\section{Roman et poésie}

Alors que les modernités américaines dans leur dimension esthétique renvoient traditionnellement à l'expérimentation formelle des artistes et auteurs des premières décennies $\mathrm{du} \mathrm{XX}^{\mathrm{e}}$ siècle et à leur relation avec l'avant-garde européenne, Céline Mansanti nous invite à laisser de côté les discours constestataires pour réfléchir à la relation entre le modernisme littéraire anglo-américain et la littérature dite mainstream de la période de l'entre-deux-guerres, qui fait actuellement l'objet de réévaluations critiques. Dans son article intitulé «Au-delà des clichés et de la satire: le débat sur l'expatriation et le modernisme dans un roman "mainstream": The French They are a Funny Race, de Lyon Mearson (1931) », Céline Mansanti pose la question du contexte de production d'une œuvre peu étudiée pour élucider des convergences d'influence entre, d'une part, le théâtre moderniste dans lequel se déroule la vie des expatriées à Paris, champ d'expérimentation réflexif qui problématise les conventions discursives de la littérature, et, d'autre part, la culture "middlebrow» ou "moyenne", où la transparence des univers fictionnel et stylistique participe des règles de la société de consommation émergente.

11 Dans « Bill \& Carlos : Les Amériques de William Carlos Williams », Anna Aublet apporte un éclairage nouveau sur la spécificité socio-culturelle de l'œuvre du poète William Carlos Williams (1983-1963), et sur la double identité linguistique et culturelle de cet auteur états-unien né d'une mère portoricaine. Prenant pour point de départ le schéma polémique de la modernité poétique, la réflexion porte sur la reconquête par la poésie de l'histoire et des mythes du continent américain, qui, chez Williams, repose sur des opérations paradoxales de déconstruction et construction. Anna Aublet étudie le réexamen des mythes fondateurs de l'histoire américaine auquel se livre Williams dans ses œuvres romanesques des années vingt, The Great American Novel (1923) et In the American Grain (1925). Les retours de l'auteur vers ses racines hispanophones participent d'une quête identitaire individuelle et collective qui traverse l'ensemble du continent, Nord et Sud. Le déchiffrement des codes sémantiques propres à Williams va de pair avec l'hypothèse d'une langue vernaculaire, démocratique mais disparate, née du métissage. Les horizons de la nouveauté, objet d'une recherche obsédante chez ce poète, s'ouvrent donc à la langue espagnole, héritage maternel et vestige du passé colonial des Antilles.

La relation au passé et aux origines des lettres nord-américaines est également au cœur de l'article d'Aurore Clavier, " "New contours suggested by old words" : la modernité américaine au tamis de l'archéologie poétique ". L'œuvre des années 1920 des poètes Wallace Stevens, Marianne Moore et William Carlos Williams, est ici raccordée à une double temporalité, à la fois rétrospective et prospective. La réflexion d'Aurore Clavier s'appuie sur les modalités archéologiques de l'invention moderniste comme paradigme d'écriture pour relier les explorations et excavations du passé à l'émergence d'un idiome poétique sensible à la dialectique entre surface et profondeur, entre les 
contingences du présent et le récit originel. Si l'entreprise historiographique de la modernité consiste à redéfinir les modes de réécriture du passé états-unien, l'identité culturelle nationale, selon Aurore Clavier, se réfère tout autant à la fascination exercée sur ces poètes par le temps long. La relation du continent américain à la modernité doit donc se lire au miroir de l'activité mytho-poétique de ses écrivains. Le déplacement qui s'opère par rapport aux points d'ancrage est tout autant d'ordre esthétique que politique et géographique, du nord au sud et de l'est vers l'ouest. Dans son approche hémisphérique, Aurore Clavier tente d'embrasser la pluralité impliquée dans l'entreprise de réécriture de l'origine, à savoir la recherche d'un présent enraciné dans des sites pluriels. Chez Williams, en particulier, le contexte historique et sociétal de In the American Grain recouvre l'Amérique centrale, les Caraïbes et les nations amérindiennes. Il permet de mettre en relief l'hypothèse d'un modernisme américain qui ne peut plus se penser comme culturellement et politiquement neutre.

Comme le rappelle François Hugonnier dans son article « Repenser les modernismes à la lueur de l'œuvre de Jerome Rothenberg ", l'avant-garde européenne avait déplacé l'accent vers les marges de la pratique artistique; ce faisant, elle ouvrait le chemin, pour la modernité américaine, vers l'hétérogénéité des formes et des cultures. La pluralité se révèle être l'un des principaux ressorts du modernisme, protéiforme et international. Lire Rothenberg (né en 1931), c'est découvrir un plaidoyer pour un nouveau théâtre de modernismes qui prend en compte, au-delà du sol américain, une myriade de sources locales et "globales» de diverses époques. François Hugonnier souligne le rôle de Rothenberg en tant que cartographe d'une poétique engagée, panaméricaine et cosmopolite, polyglotte et iconoclaste. Le militantisme éditorial de Rothenberg réalise ainsi le projet inédit de faire lire la modernité littéraire à l'aune de textes autochtones, souvent en traduction. Cet article met en valeur la pertinence du geste anthologique, compilateur des poésies sous-représentées ou enfouies sous la chape monolithique du canon occidental, en ce cinquantième anniversaire du volume pionnier que fut Technicians of the Sacred: A Range of Poetries from Africa, America, Asia, Europe \& Oceania (1968).

$14 \mathrm{Au}$ terme de ce parcours, on peut se demander, avec William Mohr, comment les mutations postmodernes ont rejailli sur la formation de communautés hétérogènes sur le continent américain, investies en tant que lecteurs, auteurs, éditeurs, imprimeurs ou critiques, dans la vie des lettres à l'époque contemporaine. Soucieux d'explorer les conditions socio-politiques de la production littéraire, avec en toile de fond la trajectoire de sa propre œuvre poétique, dans son article intitulé «La côte Ouest comme capitale littéraire dans la république de la littérature: les éditeurs indépendants comme agents plastiques de l'hyper-modernité ", William Mohr part du modèle théorique dit «circuit de communication »- proposé par l'historien américain Robert Darnton, spécialiste de l'histoire du livre - pour s'intéresser à la construction d'un canon poétique de la côte Ouest, pendant les années 1955-1985, grâce à la synergie éditoriale d'un groupe de poètes et éditeurs indépendants. Les efforts collectifs de ces acteurs culturels laissent envisager un nouveau récit de la (post-)modernité à l'aune de la dissémination culturelle. Ouvrir les lignes de démarcation d'un canon poétique, comme le fait la communauté littéraire ici célébrée par William Mohr, c'est participer aux glissements de pensée et de discours qui, jusqu'à aujourd'hui et sur différents modes, donnent la primauté au changement. 


\section{Hors Occident ?}

15 L'émergence de la modernité ne peut pas se penser sans réfléchir aux articulations entre le domaine artistique et le champ socio-politique. Pour citer encore Meschonnic, on sait que les frontières politiques et territoriales du moderne furent définies par l'Occident : «La modernité. Inutile d'ajouter: occidentale. La modernité est européenne. Et si on appelle Occident l'Europe, plus l'Amérique du Nord, elle est occidentale » (1988: 27). Mais la modernité se pose également comme «le terrain d'un travail du sens » (1988:26), sujet à la temporalité et à l'espace dans lesquels toutes les sociétés et les cultures évoluent désormais. Entrés sur ce terrain, les creusements de la critique postcoloniale ont contribué à mettre en lumière la codification occidentale européenne et américaine - qui en vient à constituer une grille de lecture homogène et universelle. À travers les formes de résistances opposées à l'uniformisation des modèles de savoir se dessine aujourd'hui un concept de «modernité » dans sa pluralité, qu'il convient de croiser à une perspective extra-occidentale.

Smaro Kamboureli interroge ainsi l'inscription de la modernité dans les cultures minorées par l'Occident à travers l'exemple de la représentation de la culture inuit de l'Igloulik, petite communauté du Nunavut dans les territoires du Nord du Canada. Dans son article intitulé "L'Opéra dans l'Arctique: Knud Rasmussen, traversées de la modernité ", se fait jour un affrontement dialogique entre deux temporalités. Entre le témoignage de l'ethnographe Knud Rasmussen de son expérience parmi les Iglulingmiurt - Du Gronland au Pacifique: deux ans d'intimité avec des tribus d'Esquimaux inconnus (trad. du danois, 1929) - et la réappropriation de ce récit et de ce moment historique par le film de Norman Cohn, Le Journal de Knud Rasmussen (2006), se profile la distance qui sépare le regard porté par l'ethnographe sur l'entreprise colonisatrice et une lecture épistémologique de la culture inuit à l'époque contemporaine. La présence de l'Occident dans les territoires du nord du Canada au début du vingtième siècle - tel l'opéra parmi les Inuits - est le signe, pour Rasmussen, de la « modernité négative ", un concept qui traduit à ses yeux le travestissement de la culture inuit. Le rapport à ce temps révolu, marqué dans le discours de l'ethnographe par la dissonance entre modernité et altérité, évolue dans le film de Cohn vers un nouveau regard, qui reconfigure le concept d' " authenticité » autochtone, en signalant à la fois les limites du régime colonial et celles de la modernité par rapport à la construction identitaire. L'enfermement dans la temporalité se voit ainsi récusé, et le film invite à lire la culture autochtone de manière diachronique, décloisonnée, loin du mythe de l'origine et du culte du «primitif ». Dans la rencontre entre l'archive occidentale et les modes de production inuit se forme une image vivante de l'expérience de l'altérité. Le récit historique revisité par le cinéma inuit s'ouvre à l'inconnu, donnant à voir la transformation exercée par l'artefact sur l'expérience de l'Autre.

Selon Susan Friedman, on entre ainsi dans le domaine de la modernité « relationnelle » (2015 : 28), un modèle sujet aux opérations des réseaux dans lesquels se confrontent des altérités et altérations radicales, toutes temporalités confondues. Au concept de modernité est venu s'adosser, dans la critique nord-américaine, le paradigme transnational pour définir les réseaux de production culturelle et ainsi dessiner une nouvelle cartographie du moderne. La mise en lumière d'une théorie diasporique de la littérature par l'« Institut TransCanada » et sa directrice Smaro Kamboureli va dans ce sens (Scandalous Bodies, 2000). De même, pour Jahan Ramazani (2009), le débat qui 
entoure l'émergence d'une "poétique transnationale" révèle l'amplitude des influences due à la porosité des frontières culturelles, depuis les avant-gardes et audelà. En déplaçant le centre vers la périphérie, cette nouvelle lecture théorique, bâtie sur un modèle d'influence dit d'enchevêtrement [ "enmeshment»], fait entendre les résonances de la culture postcoloniale dans notre perception actuelle des développements successifs de la modernité tout au long du XXe siècle et jusqu'à nos jours.

\section{BIBLIOGRAPHIE}

Casanova, Pascale, La République mondiale des lettres [1999], éd. rev. et corr., Paris, Seuil, « Points Essais », 2008.

Friedman, Susan, Planetary Modernisms: Provocations on Modernity across Time, New York, Columbia University Press, 2015.

Grenier, Catherine, «Le monde à l'envers? », in Modernités plurielles : 1905-1970, Paris, Centre Pompidou, 2013, p. 14-31.

Meschonnic, Henri, Modernité modernité, Paris, Gallimard/Verdier, « Folio Essais », 1988.

Kamboureli, Smaro. Scandalous Bodies: Diasporic Literature in English Canada. Toronto: Oxford UP, 2000.

Ramazani, Jahan, A Transnational Poetics, Chicago, University of Chicago Press, 2009.

Sarmiento, Domingo Faustino, Facundo: Civilización y barbarie [1845], Madrid, Cátedra, « Letras hispánicas ", 2006.

\section{AUTEURS}

\section{FIONA MCMAHON}

Fiona McMahon est maître de conférences en littérature américaine et directrice du Centre d'études canadiennes à l'Université de Bourgogne Franche-Comté (http://blog.u-bourgogne.fr/ cec-dijon/). Ses recherches portent sur le modernisme, les poétiques des $\mathrm{XX}^{\mathrm{e}}$ et $\mathrm{XXI}^{\mathrm{e}}$ siècles (US/ CAN), ainsi que sur des relations intermédiales dans les arts et les lettres. Parmi ses travaux sont parus deux monographies : Charles Reznikoff: une poétique du témoignage (L'Harmattan, 2011) et H.D. Trilogy (Atlande, 2013) ; elle a co-dirigé un ouvrage collectif à paraître chez Classiques Garnier en 2018, Penser le genre en poésie contemporaine et un volume à paraître en 2018 dans la revue Sillages critiques, L'archive: horizons de la création contemporaine. Fiona.McMahon@ubourgogne.fr Fiona McMahon is Associate Professor of American Literature and the director of the Center for Canadian Studies at the Université de Bourgogne Franche-Comté (Dijon, France): http://blog.ubourgogne.fr/cec-dijon/. In addition to articles on Modernist and contemporary poetry (US/ CAN), and on intermediality in the arts and literature, she is the author of Charles Reznikoff: une 
poétique du témoignage (L'Harmattan, 2010), H.D. Trilogy (Atlande, 2013) and has co-edited a book of essays, Penser le genre en poésie contemporaine (forthcoming in 2018 with Classiques Garnier) and a volume for the journal, Sillages critiques, L'archive: horizons de la création contemporaine.

Fiona.McMahon@u-bourgogne.fr

\section{PAUL-HENRI GIRAUD}

Professeur en études hispaniques contemporaines à l'Université de Lille, il est directeur adjoint du Centre d'études en civilisations, langues et littératures étrangères (CECILLE) et Secrétaire général de l'Institut des Amériques. Il a publié Octavio Paz : Vers la transparence (2002, édition en espagnol revue et augmentée 2014) et Manuel Álvarez Bravo : L'Impalpable et l'imaginaire (2012). Ces dernières années, il a notamment co-dirigé l'ouvrage 1910: México entre dos épocas (El Colegio de México, 2014) ainsi que deux dossiers monographiques : Fotografía, cultura y sociedad en América latina. Nuevas perspectivas (2015) et Cosmopolitismes dans les Amériques, 1900-1960 (2018), numéros 219 et 223 de L'Ordinaire des Amériques (http://orda.revues.org).paul-henri.giraud@univ-lille3.fr 